\title{
OPERATOR INEQUALITIES ASSOCIATED WITH TSALLIS RELATIVE OPERATOR ENTROPY
}

\section{LIMIN ZOU}

Abstract. In this paper, we present some operator inequalities related to Tsallis relative operator entropy. Our results are refinements and generalizations of some existing inequalities.

Mathematics subject classification (2010): 47A63.

Keywords and phrases: Tsallis relative operator entropy, relative operator entropy, operator inequality.

\section{REFERENCES}

[1] J. I. FUJII, E. KAMEI, Relative operator entropy in noncommutative information theory, Math. Japon., Vol. 34, 3 (1989), pp. 341-348.

[2] J. I. FUJII, E. KAMEI, Uhlmann's interpolational method for operator means, Math. Japon., Vol. 34, 4 (1989), pp. 541-547.

[3] S. FURUICHI, Inequalities for Tsallis relative entropy and generalized skew information, Linear Multilinear Algebra, Vol. 59, 10 (2011), pp. 1143-1158.

[4] S. Furuichi, K. Yanagi, K. Kuriyama, Fundamental properties of Tsallis relative entropy, J. Math. Phys., Vol. 45, 12 (2004), pp. 4868-4877.

[5] S. FURUICHI, K. YANAGI, K. KURIYAMA, A note on operator inequalities of Tsallis relative operator entropy, Linear Algebra Appl., Vol. 407, 1 (2005), pp. 19-31.

[6] T. FURUTA, Furuta's inequality and its application to the relative operator entropy, J. Operator Theory, Vol. 30, 1 (1993), pp. 21-30.

[7] T. FuRUTA, Invitation to Linear Operators, Taylor \& Francis, London and New York, (2001).

[8] T. FURUTA, Reverse inequalities involving two relative operator entropies and two relative entropies, Linear Algebra Appl., Vol. 403, 1 (2005), pp. 24-30.

[9] T. FURUTA, Two reverse inequalities associated with Tsallis relative operator entropy via generalized Kantorovich constant and their applications, Linear Algebra Appl., Vol. 412, 2-3 (2006), pp. 526-537.

[10] K. Yanagi, K. Kuriyama, S. Furuichi, Generalized Shannon inequalities based on Tsallis relative operator entropy, Linear Algebra Appl., Vol. 394, 1 (2005), pp. 109-118. 